\title{
When children forget to remember: Effects of reduced working memory availability on prospective memory performance
}

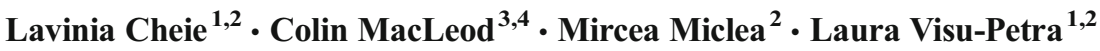

Published online: 16 December 2016

(C) Psychonomic Society, Inc. 2016

\begin{abstract}
The preparatory attentional and memory processes theory (PAM) of prospective memory (PM) proposes that prospective remembering is influenced by the variation in the availability of WM resources. Consequently, PM should be impaired when WM resources are reduced either by direct WM manipulation or by individual differences associated with restricted WM performance. Our study tested this prediction in school-age children by examining the independent and interactive effects of three factors known to deplete availability of WM resources: increased processing demands of a concurrent arithmetic task, additional WM span requirements, and high trait anxiety. A sample of 10-year-old children $(N=128)$ performed a PM task, embedded in an ongoing arithmetic task, which progressively imposed greater WM processing demands. Half of these participants also concurrently carried out an embedded WM span task. The results supported the PAM hypothesis, showing that children's PM was compromised by the restriction of WM resources, whether this resulted from increasing the processing demands on the
\end{abstract}

Lavinia Cheie

laviniacheie@psychology.ro

1 Developmental Psychology Lab, Department of Psychology, School of Psychology, Babeș-Bolyai University, Cluj-Napoca, Romania

2 Applied Cognitive Psychology Center, Department of Psychology, Babeș-Bolyai University, Cluj-Napoca, Romania

3 Centre for the Advancement of Research on Emotion, School of Psychology, University of Western Australia, Perth, Australia

4 Department of Psychology, School of Psychology, Babeș-Bolyai University, Cluj-Napoca, Romania ongoing task, from imposing additional WM span requirements, or from high trait anxiety. However, these WMdepleting factors exerted additive effects rather than an interactive impact, suggesting that they might each deplete different aspects of WM resource availability necessary for prospective remembering. Overall findings imply that children's PM success is not only associated to their WM capacity but it mostly depends upon how many of those WM resources are available to be devoted to the PM requirement.

Keywords Prospective memory $\cdot$ Working memory $\cdot$ Trait anxiety $\cdot$ Children $\cdot$ Processing demands

While retrospective memory concerns remembering past events or previously encoded information, prospective memory (PM) involves remembering to perform a previously planned action at the appropriate time or in the appropriate context (Einstein \& McDaniel, 1996; Kliegel, McDaniel, \& Einstein, 2000). In our day-to-day functioning, the vital role of prospective remembering is most vividly demonstrated when we experience PM failures, such as forgetting that we were supposed to take our medicine at 5 o'clock (time-based PM), forgetting to make an announcement during an important meeting (event-based PM), or forgetting to start working on a review after finishing reading this article (activity-based PM). Identifying and understanding the factors that influence effective event-based PM in school-age children is of crucial importance, as this is a developmental period in which everyday PM demands are likely to increase in both frequency and complexity. From the time they start school, children are required to forward plan specific activities and remember to implement them at a specific time, or in a specific context. 
Although researchers have begun to investigate children's PM abilities (Mahy, Kliegel, \& Marcovitch, 2014), relatively little is known about the factors that underpin variation in the success of children's prospective remembering.

Influential studies have converged on the hypothesis that working memory (WM) capacity is crucial for successful event-based prospective remembering (e.g., McNerney \& West, 2007; Smith, 2003; Smith \& Bayen, 2004). However, this hypothesis has not been directly evaluated in children by testing the resulting prediction that their PM performance will be impaired by factors that deplete the availability of WM resources. This study was designed to test this prediction by examining whether various sources of WM resource depletion adversely affect children's event-based PM. Specifically, we examined the independent and interactive potential impact upon children's PM of three factors known to deplete the availability of WM resources: increased processing demands, additional WM span requirements, and elevated levels of trait anxiety. Determining that children's PM is affected when their online WM resources are depleted would provide proof that not only does successful prospective remembering rely on the development of WM but it mostly depends upon how many of those WM resources can be devoted to the active maintenance of the intention to produce the PM action in the appropriate context.

\section{PM performance and availability of WM resources}

Laboratory tasks employed to investigate event-based PM functioning are designed to mimic everyday situations in which we must remember to carry out a previously planned action when an intention-relevant cue occurs, despite being in the midst of performing another activity. In the real world, for example, we might intend to interrupt reading this article and check our inbox when we receive an e-mail notification, if we are awaiting an important e-mail. Thus, in a laboratory PM task, participants are typically required to interrupt an ongoing activity in order to implement a certain action whenever a particular event occurs (e.g., Einstein \& McDaniel, 1996).

The multiprocess framework of PM (McDaniel \& Einstein, 2000) states that, under certain specific task circumstances, previously planned intentions might be retrieved automatically. Specifically, this can occur when the PM requirement is emphasized, the cue is salient, there is a high association between the cue and the PM action, and the ongoing activity implies focal processing of intention-relevant cues (McDaniel \& Einstein, 2000; McDaniel, Guynn, Einstein \& Breneister, 2004). However, according to the multiprocess framework, when such conditions are not met and PM requires active monitoring for intention-related cues, then successful PM will depend on strategic effortful resources. Likewise, the preparatory attentional and memory processes
(PAM) theory of PM (Smith, 2003; Smith \& Bayen, 2004) proposes that successful PM relies on effortful processes. However, while the multiprocess view entails that the PM intention can be spontaneously retrieved in certain conditions, the PAM theory proposes that prospective remembering always requires preparatory attentional processes. These are defined as resource demanding processes that prepare engaging resources - otherwise used for effectively responding to the ongoing task - to be enrolled to responding to the PM task, and that can also be engaged in active searching or monitoring for the intention-relevant cue (Smith, 2010). Hence, although Einstein and McDaniel (2010) argue for the existence of an automatic intention retrieval when cued in certain conditions, Smith $(2008,2010)$ argues that the occurrence of a PM cue can lead to a spontaneous capturing of attention, but not to an automatic retrieval of intention. According to the PAM framework, the preparatory attentional processes are responsible for the maintenance of intention and providing the accurate response to the PM cue. Hence, these processes are sensitive to variations in the available WM resources (Smith, 2003; Smith \& Bayen, 2005), and the more the available WM resources are restricted, the more evident the PM impairment should be. This study set out to test this hypothesis by investigating whether children's PM performance is impaired when the availability of WM resources is constrained by several factors: by increasing WM demands (via increasing processing demands and additional WM span requirements) of the concurrent tasks and by high levels of trait anxiety. If children's PM performance is affected to a greater extent by the conjunct impact of these WM resource-depleting factors, then this would provide compelling evidence that PM success is sensitive to the degree to which the availability of WM resources is reduced.

\section{Factors reducing available WM resources}

WM load WM encompasses the cognitive systems that serve to keep things in mind while performing complex operations such as reasoning, comprehension, and learning (Baddeley, 1986, 2010). Because PM success requires individuals to keep in mind the intention to produce the PM action in response to the PM cue while being engaged in a potentially complex ongoing task (Ellis \& Kvavilashvili, 2000; Smith, 2003), good intention retrieval is expected to depend upon the availability of WM resources. There is evidence to support this prediction from studies with adult participants. In some of these studies, it has been observed that the more demanding the concurrent, ongoing task is, the less likely it becomes for participants to perform the PM action when they encounter the PM cue (e.g., Einstein, Smith, McDaniel, \& Shaw, 1997; Marsh, Hancock, \& Hicks, 2002). In other studies, WM resource availability has been manipulated by requiring a conventional WM task to be performed concurrently with the PM task (Basso, Ferrari, \& Palladino, 2010; Einstein et al., 1997; Marsh \& 
Hicks, 1998; West \& Bowry, 2005). The findings indicate that, in adults, PM retrieval is significantly impaired by the concurrent WM task, especially when it involves updating verbal information (e.g., Herrmann \& Gruneberg, 1999). This suggests that aside from the central executive component of the WM system (Baddeley, 1986, 2002), PM performance may particularly be dependent on the availability of the phonological loop component. Hence, studies with adults have confirmed that the probability of their PM success decreases (1) as the difficulty of the concurrent task increases (e.g., Marsh et al., 2002), and (2) when an additional WM span (especially verbal) load is added to the concurrent task (e.g., West, Bowry, \& Krompinger, 2006). However, it is yet unknown whether these two manipulations exert an interactive impact on PM performance. Because PM success is hypothesized to be sensitive to available WM resource restrictions (Smith, 2003; Smith \& Bayen, 2005), it can also be expected that restricting the WM resources via both manipulations would exert a greater detrimental impact upon prospective remembering.

Considerably less is known about PM performance variation in children, and this is a significant omission as, during the school-age period, children are required to learn how to self-regulate their behaviors while considering their long-term plans and potential consequences (Steinberg, 2005). The school-age period is a transitional interval characterized by development in brain regions associated with central executive functioning (Giedd, 2004), as well as with event-based PM (Cheng, Wang, Xi, Niu, \& Fu, 2008). Although it is known that concurrent task load can disrupt children's PM performance (e.g., Kliegel et al., 2013; Kvavilashvili, Kyle, \& Messer, 2008, for a review), the direct implication of WM resources in children's PM functioning remains uncertain. Some studies have found that individual differences in WM capacity predict variability in children's PM performance (Mahy \& Moses, 2011; Wang, Kliegel, Liu, \& Yang, 2008). However, to our knowledge, there are no existing studies in which the putative dependency of children's PM performance on WM resource availability has been tested. Investigating children's PM variation as a function of direct manipulation of their available WM resources would shed further light into the relationship between PM and WM. This approach would determine whether children's successful prospective remembering is not only reliant on good WM capacity but also on the amount of their WM resources devoted to the active maintenance of the intention to produce the PM action. This study adopted this approach to test the hypothesis that children's PM accuracy depends upon the availability of their WM resources. As previously noted, increased WM loads can be imposed by (1) increasing processing demands, such as increasing the difficulty levels of the main arithmetic task in an Operation WM span probe (Ashcraft \& Kirk, 2001), or by (2) adding additional WM span requirements (e.g., MacLeod \& Donnellan,
1993). Both these methods were used to directly manipulate children's WM resource availability in our study, testing their PM performance while they completed an ongoing arithmetic task with increasing processing demands, with or without additional WM span requirements.

Heightened trait anxiety As a further test of the hypothesis that restricted availability of WM memory resources impairs PM functioning in children, we also evaluated the resulting prediction that PM performance would decline with increasing levels of trait anxiety. It is well established that elevated levels of trait anxiety are reliably characterized by the reduced availability of WM resources (e.g., Eysenck, Derakshan, Santos, \& Calvo, 2007; Eysenck, Payne, \& Derakshan, 2005). According to the attentional control theory (Eysenck et al., 2007), this is because anxiety-related worrisome thoughts consume WM resources, thereby disrupting performance on tasks that depend upon the central executive component of the WM system. Several studies have confirmed this trait anxiety-WM relationship in children, by showing that higher levels of trait anxiety are associated with reduced WM (e.g., Owens, Stevenson, Norgate, \& Hadwin, 2008; Ng \& Lee, 2010; Visu-Petra, Cheie, Benga, \& Alloway, 2011). Given that children with higher levels of trait anxiety display reduced availability of WM resources, the hypothesis that such resources are needed to sustain PM performance leads to the novel prediction that heightened trait anxiety in children will be related to impaired PM performance. In a recent study we found that higher trait anxiety levels were negatively related to younger, but not older, preschoolers' event-based PM performance (Cheie, Miclea, \& Visu-Petra, 2014). However, as pointed out in that study, because the task we used needed to be simple enough for the younger participants, it plausibly was too simple to tax the WM resources of the older children. The present design overcomes this problem to test, for the first time, whether PM performance in school children is impaired by high levels of trait anxiety. If indeed this is the case, then such PM impairment may represent a candidate explanatory mechanism for the well documented observation that high trait anxiety is associated with academic underachievement in children (Aronen, Vuontela, Steenari, Salmi, \& Carlson, 2005), and increased risk of social maladjustment (Weissman et al., 1999).

\section{Current study}

This study tested whether three factors known to deplete the availability of children's WM resources negatively impact their prospective remembering. Specifically, we tested whether children's PM performance was impaired by WM restrictions imposed by increasing the processing demands incurred by the ongoing arithmetic task, by varying the presence of 
additional WM span requirements and by higher levels of trait anxiety. By testing this hypothesis in children, we attempted to provide evidence showing that children's successful prospective remembering is not only associated to their WM capacity development but it mostly depends upon how many of those WM resources can be devoted to the active maintenance of intention and providing the accurate response to the PM cue. In this study, this WM-depleting requirement was undertaken by attending concurrent tasks with increasing processing demands and additional WM span requirements, and by attending anxiety-related worrisome thoughts.

On the basis of the PAM hypothesis that event-based PM success requires preparatory attentional processes that are sensitive to variations in the available WM resources (Smith, 2003; Smith \& Bayen, 2004, 2005), we predicted that the reduction of available WM resources via these three factors would lead to a decrease in children's PM performance. Based on the same premise, we predicted that the more the available WM resources are restricted, the more children's prospective remembering will be affected. Thus, we expected these WMdepleting factors to exert an interactive detrimental impact upon children's prospective remembering, such that the addition of one factor (e.g., trait anxiety) would exert a greater impact on children's PM performance when WM availability have already been depleted by one of the other two factors (i.e., increased processing demands, WM span requirements). If children's PM performance is affected to a greater extent by the interactive impact of these WM resource-depleting factors, this would provide compelling evidence for PAM's prediction (Smith, 2003; Smith \& Bayen, 2004, 2005) that PM success is sensitive to the degree to which the availability of WM resources is reduced.

\section{Method}

\section{Design overview}

We assessed children's prospective remembering while they were engaged in an ongoing arithmetic addition task. This arithmetic task was completed in three difficulty conditions, designed to impose progressively greater demands on WM resources: low, medium, and high difficulty. Each difficulty level comprised 18 sequences, each sequence delivering either two, three, or four arithmetic problems. Concurrently with this ongoing arithmetic addition task, half of the participants had to carry out only the embedded PM task (i.e., PM group), while concurrently with this embedded PM task the other half also had to carry out a WM span task, designed to further deplete available WM resources (PM + WM group). Participants were randomly assigned to either the PM group or the PM + WM group.

\section{Participants}

Parents of third- and fourth-grade children from four urban public schools in Cluj-Napoca, Romania, received a written invitation to participate in a larger scale research project aiming to evaluate children's prospective remembering and WM. Parents who gave their written parental agreement also filled in a form requiring demographic information, as well as a questionnaire regarding their child's trait anxiety level. Out of the total 280 families who received our invitation, approximately $78 \%$ parents agreed for their child to participate; and out of the total 219 remaining children, 148 were used for this particular study. Seven children were excluded from the analyses, as two had incomplete data and five failed to recall the PM task instructions. Despite random assignment of participants to the PM and the PM + WM groups, preliminary analyses indicated a group-level difference in trait anxiety. Hence, a propensity score matching procedure (Rosenbaum \& Rubin, 1983) was adopted, in which groups were balanced according to trait anxiety scores, age, and gender. This matching procedure reduced the sample size, discarding eight (out of 72) participants from the PM group and five participants (out of 69) from the PM + WM group.

Our final sample comprised 128 10-year-old children (69 girls; $M_{\text {age }}=9.97$ years; $S D=7.44$ months), assigned as follows: (1) 64 children to the PM group, (2) 64 children to the $\mathrm{PM}+\mathrm{WM}$ group. Participants assigned to the two memory groups did not differ with respect to gender distribution, $\chi^{2}(1)$ $=1.54, p=.22$, trait anxiety scores, $F(1,126)=2.66, p=.11$, or age, $F(1,126)=.13, p=.72$. All participants were monolingual children, from families with a medium to high socioeconomic status, measured as a combination of income, maternal education, and occupation indexes.

\section{Measures}

\section{Ongoing arithmetic addition task}

The ongoing arithmetic addition task was completed by all participants. It was structured to impose progressively greater demands on WM resources by sequentially delivering the low, medium, and high difficulty trials. Hence, children were first given the low difficult arithmetic problems, continued with the medium difficulty ones, and progressed to the high difficulty ones. Each arithmetic difficulty level consisted of 18 sequences of either two, three, or four arithmetic problems, presented in a textual format. Sentences appeared on the computer screen, specifying either two or three categories of items, each of which could be present in varying numbers (e.g., "For her birthday, Ana received two shirts and a ball."). Participants were instructed to read each sentence aloud, then add the total number of items present and to indicate this number verbally in response to the question asked in the final sentence appearing 
on the computer screen (e.g., "The number of things she received is..."). Children were instructed to immediately press the space bar key after presenting their answer in order to move to the next sentence.

The low difficulty condition consisted of arithmetical problems that required adding only two numbers (e.g., "For her birthday, Ana received two shirts and a ball. The number of things she received is..."). The medium difficulty condition consisted of arithmetical problems that required adding three numbers (e.g., "For her birthday, Ana received three shirts, a ball, and two sweaters. The number of things she received is..."). Finally, the high difficulty condition presented equivalent sentences to those shown in the medium difficulty condition, but required the participant to add only specified categories of items and ignore category-incompatible ones, thereby requiring categorization (e.g., "For her birthday, Ana received three shirts, a ball, and two sweaters. The number of clothes she received is..."). As WM resources underpin the successful use of categorization (see Craig \& Lewandowsky, 2012; Lewandowsky, 2011), the addition of a categorization requirement to the arithmetic task was employed to further tax children's WM resources. An overview of the arithmetic task requirements is presented in Fig. 1. Performance on this task was indexed by scoring the number of sequences on which accurate responses were given, resulting in a maximum possible score of 18 for each difficulty condition.

\section{Ongoing working memory span task}

Half of the participants also concurrently completed a WM span task. This task, which was closely modeled on the classic operation span task (e.g., Conway, Cowan, Bunting, Therriault, \& Minkoff, 2002), was intended to consume additional WM resources. The task required children, in addition to solving each of the problems presented in the arithmetic addition task, to recall results from previous arithmetic calculations. Across trials, this span task was given in three conditions that varied in terms of span length. Specifically, participants were required to recall the results to either the previous two (span list length two), three (span list length three), or four (span list length four) arithmetic problems. Each span length condition was given six times, thus composing 18 sequences ( 3 list lengths $* 6$ trials) of arithmetic problems that were presented in each difficulty condition of the ongoing arithmetic task.

Children were instructed to read aloud the arithmetic problem at their own pace, calculate the result, verbally present it to the experimenter, and immediately press the space bar to continue. After $2 / 3 / 4$ calculations, the instruction "recall the previous $2 / 3 / 4$ results" appeared, and the participants verbally reported these prior results. Therefore, to perform this span task, children were required to retain preceding calculation results in their WM, a requirement meant to further deplete the availability of WM resources.

To measure children's WM performance, we calculated an aggregate score reflecting WM performance on all span lists. In this respect, we first scored each list length at which all six trials were passed correctly (e.g., a score of 2 for all trials passed in the span list length two, a score of 3 for all trials passed in the span list length three); then a score of 0.17 (i.e. 1/6) was added for each item that was recalled correctly from the following list. Thus, if a child correctly recalled all six trials of two addition results (span list length two), six trials of three addition results (span list length three) and three trials of four addition results (span list length four) a score of $2+3+$ $(3 * 0.17)=5.51$ was awarded.

\section{Prospective memory task}

All participants were asked to fulfil a PM requirement while carrying out the ongoing arithmetic addition task. This PM task was modeled after the laboratory procedure employed by Einstein and McDaniel (1990). It required children to remember to refrain from calculating the addition result, and to instead press the Enter key, as soon as they detected the word ball within any sentence (see Fig. 1 for task illustration). Thus, effective PM performance required participants to monitor for the intend-related cue, inhibit normal activity upon encountering it, and instead engage in the alternative action.

The PM cue appeared on three out of the 18 sequences of arithmetic problems pertaining to each difficulty condition (low, medium, high difficulty) of the ongoing task. Hence, the PM cue appeared in a total of nine such sequences (out of 54) throughout the ongoing task. In addition to ensuring that the PM cue appeared three times in each arithmetic difficulty condition, we also ensured that within each difficulty condition, it appeared once in each of the three list lengths (of 2/3/4 elements) in the WM span task. PM performance was scored by awarding one point each time the correct action was performed in response to the PM cue. Thus, the maximum score achievable in each ongoing arithmetic difficulty condition was 3.0. A response was counted as correct only when children refrained from saying the arithmetic calculation result and also pressed the Enter key prior to the onset of the subsequent trial.

\section{Trait anxiety measure}

The Romanian version of the Revised Child Anxiety and Depression Scale (RCADS; Chorpita, Yim, Moffitt, Umemoto, \& Francis, 2000) was used to assess trait anxiety in children. The RCADS contains 47 items referring to the frequency of occurrence of different anxiety and depression symptoms. Parents are asked to rate the frequency of occurrences on a 4-point scale, where $0=$ never and $4=$ always. The 


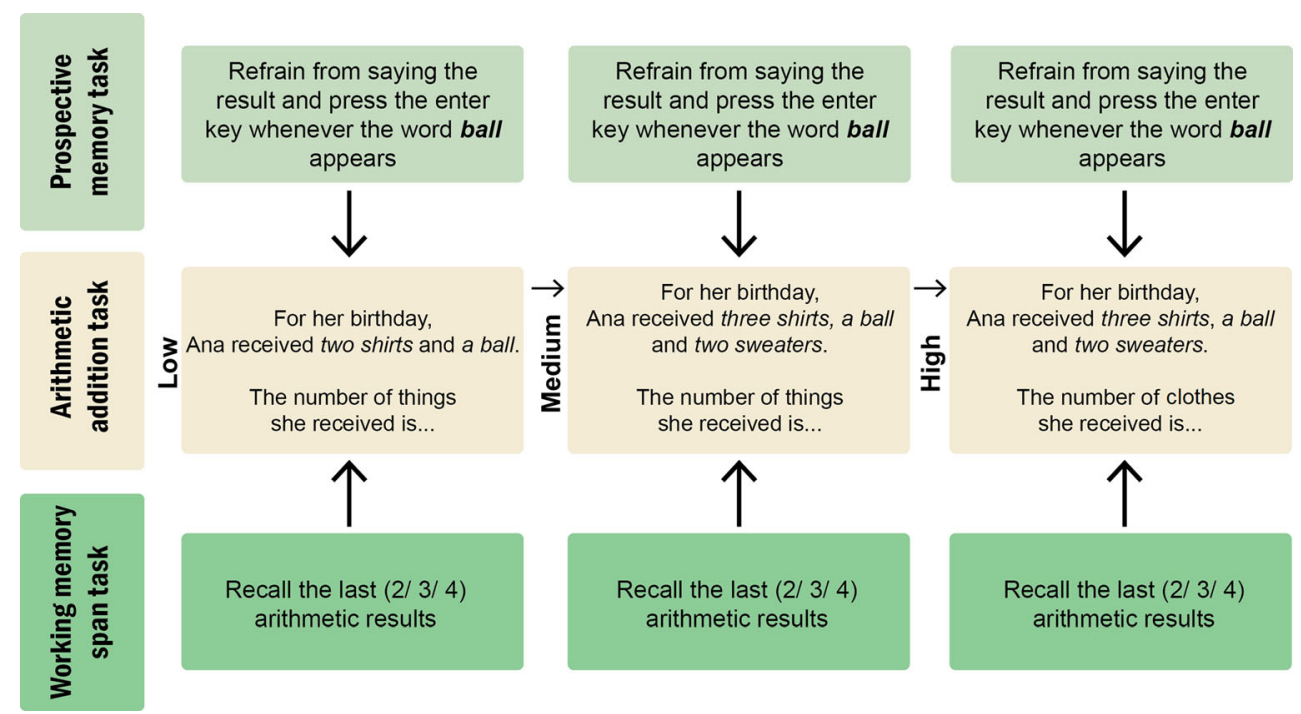

Fig. 1 Illustration of the ongoing arithmetic addition task with its three increasing processing conditions (low, medium, high difficulty) and concurrent memory requirements, either prospective memory (PM) or

RCADS contains six subscales, five of which tapping specific aspects of child anxiety. The overall score on the five anxiety domains provides a general trait anxiety measure, which has been found to be a significant indicator of DSM-IV anxiety dysfunction (e.g., Nauta et al., 2004). The internal consistency of the RCADS trait anxiety scores is very high (Cronbach's alpha $=0.91$; Ebesutani et al., 2010) and its DSM-IV consistent factor structure was validated in both clinical and school samples (Ebesutani et al., 2011; Ebesutani et al., 2010). This Romanian version has been found to be a valid and reliable measure of trait anxiety in Romanian school children (incaș, Cheie, Mărcuș, Benga, \& Visu-Petra, 2016), and high internal consistency for trait anxiety scores was also found in the current sample (Cronbach's alpha $=0.87)$.

\section{Procedure}

Children were individually tested in a single session, carried out in a separate room at their school. Three research assistants were trained to administrate the tasks. As we first had access to only two schools, children in both schools (58\% of the final sample) were randomly assigned to one of the two memory groups. For the other two schools, we again had the same research assistants and children in both schools were randomly assigned to one of the memory groups.

The ongoing arithmetic addition task was identical across memory groups (PM group vs. PM + WM group) and, along with the assigned memory requirement, was administered in a computerized format (designed using the E-Prime software, Version 1.2; Psychology Software Tools). The experimenter first provided instructions for the ongoing arithmetic task and then presented two practice trials in which children were required to read aloud the sentences, add the total number of working memory (WM). All children performed the arithmetic addition task with increasing processing demands and concurrently had either a PM task or a combined PM + WM task

elements present within the sentence, give the response to the experimenter, and press the space key immediately after. Next, the experimenter provided instructions for the WM span task to participants in the PM + WM group. After informing these participants how to perform this WM span task, they were presented with three further practice trials in which they had to carry out this task by recalling (and telling the experimenter) the last three arithmetic results. After ensuring that the participants understood the ongoing task rules, the experimenter then provided instructions for the PM component. Children were instructed how to perform the PM action when the cue appeared within a sentence, then were presented with three additional practice trials, one of which contained the cue. Participants then performed the full task in their assigned condition. To reduce fatigue, participants had 5-minute breaks after every 18 sequences of 2/3/4 arithmetic problems. At the end of the session, children were praised for their performance and rewarded with a wooden cartoon pen for their effort. The reward was not discussed before the actual testing.

After task completion, children who failed to respond to any of the PM cues, were asked a series of questions meant to establish whether the lack of a PM response was a retrospective memory failure to remember the initial instructions. They were asked the three questions recommended by Kvavilashvili et al. (2008) to test whether they could recall the PM task instructions. Five children failed to demonstrate remembering the PM requirement, and so they were excluded from the sample.

\section{Results}

Table 1 summarizes mean central tendencies in each experimental group in terms of children's age, trait anxiety scores, 
Table 1 Means (and standard deviations) for study measures, as a function of processing demands imposed by the arithmetic addition conditions (low, medium, and high difficulty), and concurrent memory task requirements (PM group or PM + WM group)

\begin{tabular}{lll}
\hline & Prospective memory group $(n=64)$ & Prospective \& working memory group $(n=64)$ \\
\hline Age (in years) & $10.0(6.11$ months $)$ & $9.9(8.60$ months $)$ \\
Trait anxiety & $21.39(9.20)$ & $18.63(9.94)$ \\
Prospective memory performance in each arithmetic addition condition & \\
$\quad 1.89(1.30)$ & $1.59(1.31)$ \\
$\quad$ Low difficulty & $1.77(1.37)$ & $1.27(1.30)$ \\
Medium difficulty & $1.56(1.39)$ & $1.20(1.32)$ \\
High difficulty & $15.74(2.80)$ & $16.37(1.98)$ \\
Ongoing arithmetic addition performance in each condition & $15.70(1.66)$ \\
$\quad$ Low difficulty & $14.73(2.94)$ & $15.40(1.39)$ \\
Medium difficulty & $14.35(4.06)$ & \\
High difficulty & - & $3.72(2.54)$ \\
Working memory span performance in each arithmetic addition condition & $2.60(2.12)$ \\
Low difficulty & - & $2.32(1.99)$ \\
Medium difficulty & - & \\
High difficulty & &
\end{tabular}

and their task performances (PM, ongoing arithmetic addition task, and WM) as a function of the processing demands imposed by the ongoing arithmetic addition conditions (low, medium, and high difficulty).

\section{Children's PM performance}

Linear mixed models were employed to determine whether there were independent effects of the WM resource-depleting factors (increased arithmetic difficulty, additional WM span requirements, and higher levels of trait anxiety) upon children's PM, as well as to test for their potential interactions. Hence, PM scores were submitted to a 2 (memory task condition: PM vs. $\mathrm{PM}+\mathrm{WM}) \times 3$ (arithmetic task difficulty: low, medium, high) linear mixed model, with repeated measures on the latter factor and trait anxiety scores treated as a continuous covariate. An unstructured covariance structure was used across arithmetic difficulty conditions, based on the prediction of a nonsystematic pattern of covariance and goodness-of-fit indices (Field \& Wright, 2011). Random intercept models were added comprising variation across participants and schools children were attending. An unstructured covariance was assumed. The effects found in the main analytical model showed nonsignificant variances in random intercepts across participants and schools, $\operatorname{Var}\left(u_{0 j}\right)=.53, \chi^{2}(10)=2.12, n s$.

First, the results revealed a significant main effect of memory task condition, reflecting the fact that children who performed the concurrent WM span task were less successful in implementing the PM intention $\left(M_{P M+W M}=1.31\right.$, $S E=.15)$ than were children who did not have this concurrent WM requirement $\left(M_{P M}=1.79, S E=.15\right), F(1,127)=5.41$, $p=.022, b=-.48, S E=.20,95 \% \mathrm{CI}[-.90,-.07]$. This supports the prediction that the consumption of WM resources by this concurrent span task would impair children's PM performance. There was also a significant main effect of arithmetic task difficulty, $F(2,126)=10.91, p<.001$, reflecting the fact that PM accuracy was reduced by increasing arithmetic task difficulty. Thus, children's PM accuracy scores were significantly lower in the high difficulty condition than in the medium difficulty condition $\left(M_{\text {high }}=1.39\right.$, $S E=.11$ vs. $M_{\text {medium }}=1.53, S E=.12 ; b=-.14, S E=.07$, $p=.05,95 \%$ CI $[-.27, .00])$, and significantly lower in the medium difficulty condition than in the low difficulty condition $\left(M_{\text {medium }}=1.53, S E=.12\right.$ vs. $M_{\text {low }}=1.74, S E=.11$; $b=-.22, S E=.07, p=.003,95 \%$ CI $[--.36,-.07])$. This supports the prediction that the depletion of WM resources resulting from increased difficulty of the arithmetic task would impair children's PM performance.

Finally, there was also a significant main effect of trait anxiety, $F(1,127)=17.00, p<.001$, reflecting the fact that PM accuracy was reduced as a function of increasing trait anxiety, $b=-.04, S E=.01, t(127)=-4.12, p<.001$, $95 \%$ CI $[-.07,-.02]$. This supports the prediction that the restriction of WM resources associated with higher levels of trait anxiety would impair PM performance. Interactions between arithmetic task difficulty, the presence of additional WM span requirements, and levels of trait-anxiety were all nonsignificant (all $p \mathrm{~s} \geq .26$ ), reflecting a lack of conjunct impact of these WM resource-depleting factors.

Figure 2 depicts children's PM performance variation as a function of each of these WM resource depleting factors. 
Figure 2a shows children's PM performance as a function of the memory task condition and arithmetic task difficulty, whereas Fig. 2b depicts their PM performance as a function of trait anxiety scores.

\section{Children's performance on the ongoing concurrent tasks}

\section{Ongoing arithmetic addition task}

Children's performance on the ongoing arithmetic addition task was analyzed as a function of the task's difficulty conditions (low, medium, high difficulty) and the memory task concurrently performed (PM vs. PM + WM). Thus, arithmetic addition scores were submitted to a linear repeated measures mixed model where the difficulty condition was the within factor, and the memory task condition was the between variable. An unstructured covariance structure was used across arithmetic difficulty conditions. First, as expected, the results revealed that children's arithmetic performance significantly decreased as the arithmetic difficulty increased, $F(2,127)=13.24, p<.001$. Specifically, children's arithmetic performance was, on average, 1.18 points higher when the task was given in the low difficulty condition that when it was given in the high difficulty condition $\left(M_{\text {low }}=16.05, S E=.22\right.$ vs. $M_{\text {high }}=14.87, S E=.27$, $p<.001,95 \%$ CI $[.63, .1 .74])$. Second, children assigned to the $\mathrm{PM}+\mathrm{WM}$ group had a significantly better arithmetic performance $\left(M_{P M+W M}=15.80, S E=.27\right)$ compared to children assigned to the PM only group $\left(M_{P M}=14.96, S E=.27\right)$, $F(1,126)=5.00, p=.027, b=.84, S E=.37,95 \% \mathrm{CI}$ $[.10,1.58]$. The interaction between difficulty condition and memory task was nonsignificant $(p=.63)$, reflecting the fact that children's arithmetic performance was not more impaired by the increasing difficulty in one memory task condition compared to the other. Means and standard deviations for each variable are presented in Table 1.

\section{Ongoing working memory span task}

Linear mixed models for repeated measures were employed with arithmetic task difficulty introduced as the fixed repeated measures factor (low, medium, and high difficulty conditions) and trait anxiety as the fixed-factor covariate. Again, an unstructured covariance structure was used across arithmetic difficulty conditions, based on the prediction of a nonsystematic pattern of covariance and goodness-of-fit indices (Field \& Wright, 2011). As expected, there was a significant main effect of arithmetic task difficulty, $F(2,63)=14.93, p<.001$, reflecting a reduction in children's WM span as arithmetic problem difficulty increased. Thus, children's WM performance was, on average, 1.40 points higher when the concurrent arithmetic task was given in the low difficulty condition that when it was given in the high difficulty condition $\left(M_{\text {low }}=3.72, S E=.32\right.$ vs. $M_{\text {high }}=2.32, S E=.25, p<.001$, $95 \%$ CI $[.86,1.95])$. This supports the assumption that increasing arithmetic task difficulty imposed an increasing WM load. Also, trait anxiety in interaction with arithmetic task difficulty had a detrimental impact on children's WM spans, $F(2,62)=3.33, p=.042$. Analyses revealed that children with higher trait anxiety scores were significantly more impaired in the high difficulty condition compared to the medium difficulty one, $b=-.06, S E=.02, t(63)=-2.31, p=.024$, $95 \%$ CI [-..01, -.10], but not significantly more impaired in the medium difficulty condition compared to the low difficulty one $(p=.15)$. Hence, results showed that higher levels of trait anxiety affected children's WM span when their WM resources were already taxed by the demands of the ongoing arithmetic task delivered in its most difficult condition.
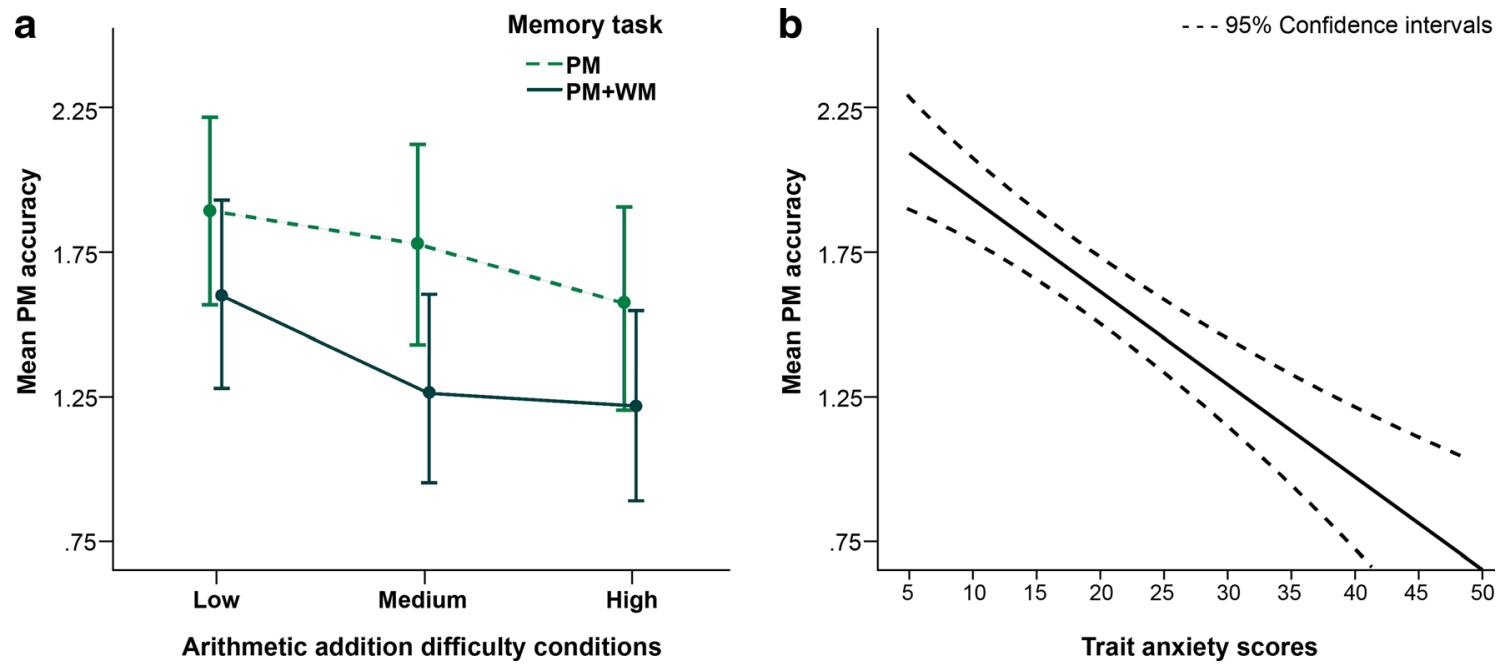

Fig. 2 Mean prospective memory (PM) accuracy scores according to memory task condition (PM vs. PM + WM) and arithmetic addition difficulty conditions: low, medium, high difficulty (a), and as a function of children's trait anxiety scores (b) 


\section{Discussion}

Primarily based on predictions generated by the PAM theory of PM (Smith, 2003; Smith \& Bayen, 2004, 2005), according to which the realization of delayed intentions is supported by preparatory processes sensitive to variations in available WM resources, we predicted that depleting the availability of WM resources will lead to a decrease in school children's PM performance. To test this assumption, we introduced three factors known to influence the availability of WM resources: increased processing demands incurred by an arithmetic ongoing task, additional WM span requirements, and high trait anxiety. Our results support the predictions generated by the PAM theory concerning the individual impact of these three factors on children's intention retrieval. Specifically, when WM resources were depleted by any of the three factors, children's PM performance was impaired. Thus, children's PM performance was hindered by greater processing demands of the ongoing arithmetic task, by additional WM maintenance requirements, and by higher trait anxiety levels. Importantly, however, the three factors exerted an additive impact on PM performance, rather than an interactive effect. Hence, the addition of one of these extra WM resource depleting factors did not exert a disproportionately great impact on PM performance when WM availability already had been depleted by one of the other factors.

Consistent with the results of previous studies showing that PM failures in adults are associated with limited WM (Basso et al, 2010; Kidder, Park, Hertzog, \& Morrell, 1997; Kliegel \& Jäger, 2006; Logie, Cocchini, Delia Sala, \& Baddeley, 2004), children's prospective remembering was adversely affected by the depletion of their available WM resources. Importantly, in this study, PM performance was found to be impaired not only by a concurrent WM span task but also by the increasing processing demands of the ongoing arithmetic task. Hence, even in the absence of a concurrent WM span task, the increased consumption of WM resources by greater ongoing task demands served to reduce PM performance. These findings support the hypothesis that good WM capacity is associated with children's PM (e.g., Mahy, Moses, \& Kliegel, 2014), but also demonstrate that children's successful prospective remembering relies on these WM resources to be available and not engaged in responding to a WM-depleting concurrent task. Thus, finding that children's prospective remembering is impaired by depleting their available WM resources, either through imposing an additional WM span task or by increasing the processing demands of the ongoing task, indicates that PM relies heavily on the availability of WM resources to actively maintain the PM intention and successfully monitor for intention-relevant cues.

Further support for the hypothesis that PM performance depends on WM availability was provided by our finding that children with higher trait anxiety levels had significantly more
PM failures. It is well-established that higher trait anxiety levels are characterized by depleted WM availability, both in adults (Eysenck et al., 2007; Eysenck et al., 2005), and in children (e.g., Owens et al., 2008; Visu-Petra et al., 2011), potentially as a consequence of worrying interfering with the active maintenance of task-related goals (Eysenck et al., 2007). Hence, the present demonstration that higher levels of trait anxiety in school-age children is associated with poorer PM is consistent with the premise that these children's PM functioning is dependent on the availability of WM resources. To our knowledge, this represents the first evidence that prospective remembering in school-age children is adversely affected by heightened trait anxiety, which we have found to impact PM in the same way as does direct depletion of WM resources through concurrent load manipulations. This adds to our knowledge of how trait anxiety can compromise cognitive functioning in children.

The findings of this study, showing that children's prospective remembering is impaired when available WM resources are reduced either by increasing WM processing demands or additional WM span requirements, or by high levels of trait anxiety, are compatible with both the PAM theory (Smith, 2003; Smith \& Bayen, 2004) and the multiprocess framework of PM (McDaniel \& Einstein, 2000). They demonstrate that when intention retrieval requires active monitoring of the cues, prospective remembering relies on effortful cognitive resources. However, motivated by the idea that the realization of delayed intentions is supported by preparatory processes that are sensitive to variations in available WM resources (Smith, 2003; Smith \& Bayen, 2005; Smith et al., 2007), children's PM performance was expected to be affected to a greater extent by the interactive impact of these WM resource-depleting factors. Yet the three factors introduced to deplete WM resources did not exert an interactive impact on PM performance. Thus, the addition of one of these factors did not exert a greater negative impact on PM performance when WM availability already had been depleted by one or both of the other two factors. While this finding seems at odds with the idea that prospective remembering success should be less probable as more available WM resources are restricted, it cannot be considered a result that invalidates PAM's premise. In this respect, our findings already confirmed that children's prospective remembering becomes less successful as their WM resources are progressively captured by a WM demanding concurrent task. For instance, the results showed that the more WM processing demands were imposed by the ongoing arithmetic addition task (low, medium, high difficulty conditions), the less successful children were in their PM performance. This provides evidence for the hypothesis that the realization of intentions is supported by preparatory processes which are sensitive to variations in available WM resources (Smith, 2003; Smith \& Bayen, 2005).

A possible explanation for the lack of an interactive detrimental effect of the three WM-depleting factors is that these 
factors might not deplete the same pool of WM resources needed for a successful realization of intention. Hence, high trait anxiety, increased processing demands of the ongoing arithmetic task, and the presence of a concurrent WM span task might each follow a distinctive pathway to affect prospective remembering by depleting different types of WM resources. In line with this explanation, there is some prior evidence that the three factors investigated here might solicit different WM components (Baddeley, 1986, 2002). Specifically, the detrimental effects of trait anxiety have been primarily observed on tasks soliciting the central executive component of WM (see Visu-Petra, Cheie, \& Mocan, 2013), arithmetic addition task performance has been shown to rely heavily on the phonological loop component of WM (e.g., Fürst \& Hitch, 2000), whereas WM span tasks involving digits additionally rely on the visuospatial sketchpad component of WM thought to be involved in the visual representation of the to be remembered numbers (e.g., Hoshi et al., 2000). If indeed the three factors each deplete a different WM resource, then it follows from the observation that good PM functioning must depend on a broad range of WM resources, plausibly including the central executive, the phonological loop, and the visuospatial sketchpad components of the WM system. Further research is needed to shed light on the pathways through which the three factors exert their impact upon children's prospective remembering, but the negative impact of WM resource depletion on children's PM performance indicates that the availability of WM resources contributes to children's PM success. Of course, good PM performance is also associated to many types of cognitive processes, including cognitive flexibility (Mahy \& Munakata, 2015), inhibitory control (Wang et al., 2008), monitoring abilities, or episodic future thinking (Nigro, Brandimonte, Cicogna, \& Cosenza, 2014). Thus, a potential direction for future research is to examine how the current WM-depleting factors interact with such processes to sustain successful realization of intentions. These interactions can shed further light regarding the unique pathways leading to prospective remembering failures. For instance, recent studies have found evidence that elevated trait anxiety also negatively impacts children's cognitive flexibility (Mărcuş, Stanciu, MacLeod, Liebregts, \& Visu-Petra, 2015). Hence, reduced cognitive flexibility may also contribute to high trait anxious children's reduced prospective remembering, as successful PM would require active maintenance of the intention while searching for the intention-relevant cues, as well as flexible alternation between responding to the ongoing task and responding to the PM one. Future studies could directly examine this hypothesis by examining the potential role of WM and cognitive flexibility in the relationship between heightened trait anxiety and PM failures.

Children's ongoing performance in the arithmetic addition task decreased as a result of the increasing processing demands of the task, demonstrating that difficulty increased as a function of task manipulations. However, a counterintuitive finding was the fact children's arithmetic performance was actually superior in the $\mathrm{WM}+\mathrm{PM}$ condition, compared to their performance on the PM condition where the additional WM requirement was absent. In this respect, previous research shows that the relationship between available WM resources and individuals' performance on concurrent tasks competing for available attentional/WM resources is not straightforward. Specifically, accuracy on the ongoing task is not necessarily expected to decrease as a consequence of WM resource depletion, as performance in this case could be the outcome of how participants assess the difficulty in carrying out both tasks concurrently (see Hicks, Marsh, \& Cook, 2005). If individuals assess that it would be difficult to complete both tasks successfully, they might emphasize one task by allocating resources toward it, to the detriment of the other. Thus, in our case, if children's performance on the ongoing task was actually superior when the WM span component was present, this could be the outcome of them allocating more resources toward it, compared to those allocated to the WM span task. Similarly, in one of Smith's experiments (2003, Exp. 3) it has been shown that although individuals with high WM capacity are more likely to be successful on the PM task performed when ongoing task demands are increased, those with low WM capacity also have comparable PM performances if they shift their resources from the ongoing task. Hence, when the two tasks that are concurrently performed compete for available WM resources, it seems that the performance on the two tasks depends on the proportion of available resources that individuals choose to allocate to one or the other. Nevertheless, the fact that we did not have a measurement of arithmetic addition in the absence of a secondary memory task (either PM or PM + WM combined) preclude us from drawing strong conclusions regarding children's arithmetic performance.

Results regarding children's WM scores revealed a detrimental impact of increased arithmetic processing demands upon children's WM spans, and that this impact was greater when children's levels of anxiety were higher. These results confirm that WM available resources needed for successful PM are reduced not only by performing a task with a WM span requirement but also by completing an increasingly processing-demanding arithmetic task (see also Adams \& Hitch, 1997; DeStefano \& LeFevre, 2004), and by having heightened trait anxiety (see also Owens et al., 2008; Visu-Petra et al., 2011).

Some limitations of the study should be acknowledged. First, there was no condition in which WM would not be, to some extent, taxed by ongoing task demands. Hence, even without a concurrent WM span task and in the absence of heightened trait anxiety, the easiest arithmetic task condition will still have made some demands upon WM resources, potentially with a detrimental impact upon children's PM. 
However, our hypothesis was tested by examining whether manipulations designed to further increase the depletion of WM would detrimentally affect children's PM performance. WM is a limited capacity system that operates as "mental workspace" in which information is being both processed and maintained (e.g., Engle, Cantor, \& Carullo, 1992). Hence, activities that load heavily on either processing or storage components are likely to place excessive demands on limited resources and result in WM resource depletion (Gathercole, Lamont, \& Alloway, 2006). Second, even though children had breaks between conditions and did not report being tired at the end of the testing session, fatigue could have compromised performance on the PM task. Conceivably, the conditions in which WM resources were most heavily taxed may have given rise to the greatest fatigue, and so PM impairment in these conditions could potentially reflect the impact of greater fatigue. Third, overall performance on the arithmetic addition task was close to ceiling levels, raising the possibility that it may not have been challenging enough to have severely taxed these children's WM resources. Nevertheless, increasing the difficulty level of the task did impact children's performance on the concurrent PM task in the expected direction. At the same time, children's mean PM scores and standard deviations in the most demanding conditions (i.e., medium and high difficulty conditions of the PM + WM task) also indicate that performance in these conditions was closer to floor levels, suggesting that some children were potentially overwhelmed by the increasing WM-taxing requirements. Although increasingly taxing the WM resources did significantly differentiate between children's PM performance in these conditions, the fact that their scores were closer to floor levels might indicate that the task was too demanding to accurately assess some 10 -year-olds' prospective remembering. Future studies should take this constraint into account, either by tailoring the WM resource depletion variations in accordance to each child's previously measured WM capacity, or controlling for their WM abilities measured in absence of a PM requirement.

These limitations notwithstanding, our findings confirm PAM's premise that the realization of delayed intentions is supported by preparatory processes which are sensitive to variations in available WM resources (Smith, 2003; Smith \& Bayen, 2005). Specifically, our results showed that children's PM was compromised by the restriction of WM resources, whether this resulted from increasing processing demands by the ongoing task, from imposing additional WM span requirements, or from high trait anxiety. These findings not only confirm that children's PM performance is associated to their WM capacity but also show that successful prospective remembering mostly depends upon how many of those WM resources can be devoted to the PM requirement, and not undertaken by attending to concurrent WM-depleting requirements, and by attending to anxiety-related worrisome thoughts. Additionally, results on the concurrent arithmetic task (i.e., children's arithmetic performance was actually superior when an additional WM requirement was present) also suggest that if individuals assess that it would be difficult to complete both tasks successfully, then they might emphasize one task by allocating resources toward it, to the detriment of the other. Taken together, findings indicate that how children choose to allocate their WM resources to one task over the other is actually more important for PM success than how well their WM capacity is developed. At the same time, this study provides first evidence that prospective remembering in school-age children is adversely affected by an affective dimension known to reflect individual differences in WM performance, heightened trait anxiety. This was found to impact on PM in the same way as does direct depletion of WM resources through concurrent load manipulations, hence also confirming PAM theory's assumption that PM success depends upon the availability of WM resources. Therefore, our study provides evidence that children's successful prospective remembering relies heavily on the availability of WM resources, potentially needed to actively maintain the PM intention to be implemented when cued and successfully monitor for the intention-relevant cues. Importantly, our observation that high processing demands of the ongoing task, additional WM span requirements, and high trait anxiety levels exerted an additive, rather than an interactive detrimental impact upon children's PM performance suggests that each of these three factors deplete different aspects of WM resources availability, all of which are necessary to enable PM success.

Acknowledgements This work was supported by a grant from the Romanian National Authority for Scientific Research, CNCSUEFISCDI, project number PNII-ID-PCCE-2011-2-0045 and by a grant from the Romanian National Authority for Scientific Research, CNCSUEFISCDI, project number PN-II-RU-TE-2012-3-0323.

The authors are grateful to the children, parents, and schools for their involvement in the study.

\section{References}

Adams, J. W., \& Hitch, G. J. (1997). Working memory and children's mental addition. Journal of Experimental Child Psychology, 67(1), 21-38.

Aronen, E. T., Vuontela, V., Steenari, M. R., Salmi, J., \& Carlson, S. (2005). Working memory, psychiatric symptoms, and academic performance at school. Neurobiology of Learning and Memory, 83(1), $33-42$.

Ashcraft, M. H., \& Kirk, E. P. (2001). The relationships among working memory, math anxiety, and performance. Journal of Experimental Psychology: General, 130(2), 224-237.

Baddeley, A. D. (1986). Working memory. Oxford, UK: Clarendon Press.

Baddeley, A. D. (2002). Is working memory still working? European Psychologist, 7(2), 85-97.

Baddeley, A. (2010). Working memory. Current Biology, 20(4), R136R140. 
Basso, D., Ferrari, M., \& Palladino, P. (2010). Prospective memory and working memory: Asymmetrical effects during frontal lobe TMS stimulation. Neuropsychologia, 48(11), 3282-3290.

Cheie, L., Miclea, M., \& Visu-Petra, L. (2014). What was I supposed to do? Effects of individual differences in age and anxiety on preschoolers' prospective memory. International Journal of Behavioral Development, 38(1), 52-61.

Cheng, H. D., Wang, K., Xi, C. H., Niu, C. S., \& Fu, X. M. (2008). Prefrontal cortex involvement in the event-based prospective memory: Evidence from patients with lesions in the prefrontal cortex. Brain Injury, 22(9), 697-704.

Chorpita, B. F., Yim, L. M., Moffitt, C. E., Umemoto, L. A., \& Francis, S. E. (2000). Assessment of symptoms of DSM-IV anxiety and depression in children: A Revised Child Anxiety and Depression Scale. Behaviour Research and Therapy, 38, 835-855.

Conway, A. R. A., Cowan, N., Bunting, M. F., Therriault, D., \& Minkoff, S. (2002). A latent variable analysis of working memory capacity, short term memory capacity, processing speed, and general fluid intelligence. Intelligence, 30, 163-183.

Craig, S., \& Lewandowsky, S. (2012). Whichever way you choose to categorize, working memory helps you learn. The Quarterly Journal of Experimental Psychology, 65(3), 439-464.

DeStefano, D., \& LeFevre, J. A. (2004). The role of working memory in mental arithmetic. European Journal of Cognitive Psychology, 16(3), 353-386.

Ebesutani, C., Bernstein, A., Martinez, J. I., Chorpita, B. F., \& Weisz, J. R. (2011). The Youth Self Report: Applicability and validity across younger and older youths. Journal of Clinical Child \& Adolescent Psychology, 40(2), 338-346.

Ebesutani, C., Bernstein, A., Nakamura, B. J., Chorpita, B. F., HigaMcMillan, C. K., Weisz, J. R., \& Research Network on Youth Mental Health. (2010). Concurrent validity of the Child Behavior Checklist DSM-oriented scales: Correspondence with DSM diagnoses and comparison to syndrome scales. Journal of Psychopathology and Behavioral Assessment, 32(3), 373-384.

Einstein, G. O., \& McDaniel, M. A. (1990). Normal aging and prospective memory. Journal of Experimental Psychology: Learning, Memory, and Cognition, 16, 717-726.

Einstein, G. O., \& McDaniel, M. A. (1996). Prospective memory: Remembering a forgotten topic. In D. Hermann, C. McEvoy, C. Hertzog, P. Hertel, \& M. Johnson (Eds.), Basic and applied memory research: Practical applications (Vol. 2, pp. 79-94). Mahwah, NJ: Erlbaum.

Einstein, G. O., \& McDaniel, M. A. (2010). Prospective memory and what costs do not reveal about retrieval processes: A commentary on Smith, Hunt, McVay, and McConnell (2007). Journal of Experimental Psychology: Learning, Memory, and Cognition, 36(4), 1082-1088.

Einstein, G. O., Smith, R. E., McDaniel, M. A., \& Shaw, P. (1997). Aging and prospective memory: The influence of increased task demands at encoding and retrieval. Psychology and Aging, 12(3), 479-488.

Ellis, J., \& Kvavilashvili, L. (2000). Prospective memory in 2000: Past, present, and future directions. Applied Cognitive Psychology, 14, 1-9.

Engle, R. W., Cantor, J., \& Carullo, J. J. (1992). Individual differences in working memory and comprehension: A test of four hypotheses. Journal of Experimental Psychology: Learning, Memory, and Cognition, 18(5), 972-992.

Eysenck, M. W., Derakshan, N., Santos, R., \& Calvo, M. G. (2007). Anxiety and cognitive performance: The attentional control theory. Emotion, 7, 336-353.

Eysenck, M., Payne, S., \& Derakshan, N. (2005). Trait anxiety, visuospatial processing, and working memory. Cognition \& Emotion, 19(8), 1214-1228.

Field, A. P., \& Wright, D. B. (2011). A primer on using multilevel models in clinical and experimental psychopathology research. Journal of Experimental Psychopathology, 2(2), 271-293.
Fürst, A. J., \& Hitch, G. J. (2000). Separate roles for executive and phonological components of working memory in mental arithmetic. Memory \& Cognition, 28(5), 774-782.

Gathercole, S. E., Lamont, E., \& Alloway, T. P. (2006). Working memory in the classroom. Working Memory and Education, 219-240.

Giedd, J. N. (2004). Structural magnetic resonance imaging of the adolescent brain. Annals of the New York Academy of Sciences, 1021(1), 77-85.

Herrmann, D. J., \& Gruneberg, M. M. (1999). How to cure your memory failures. London, UK: Blandford.

Hicks, J. L., Marsh, R. L., \& Cook, G. I. (2005). Task interference in timebased, event-based, and dual intention prospective memory conditions. Journal of Memory and Language, 53(3), 430-444.

Hoshi, Y., Oda, I., Wada, Y., Ito, Y., Yamashita, Y., Oda, M.,...\& Tamura, M. (2000). Visuospatial imagery is a fruitful strategy for the digit span backward task: A study with near-infrared optical tomography. Cognitive Brain Research, 9(3), 339-342.

Kidder, D. P., Park, D. C., Hertzog, C., \& Morrell, R. W. (1997). Prospective memory and aging: The effects of working memory and prospective memory task load. Aging, Neuropsychology, and Cognition, 4(2), 93-112.

Kliegel, M., \& Jäger, T. (2006). The influence of negative emotions on prospective memory: A review and new data. International Journal of Computational Cognition, 4, 1-17.

Kliegel, M., Mahy, C. E., Voigt, B., Henry, J. D., Rendell, P. G., \& Aberle, I. (2013). The development of prospective memory in young schoolchildren: The impact of ongoing task absorption, cue salience, and cue centrality. Journal of Experimental Child Psychology, $116(4), 792-810$

Kliegel, M., McDaniel, M. A., \& Einstein, G. O. (2000). Plan formation, retention and execution in prospective memory: A new approach and age-related effects. Memory \& Cognition, 28, 1041-1049.

Kvavilashvili, L., Kyle, F., \& Messer, D. J. (2008). Prospective memory in children: Methodological issues, empirical findings and future directions. In M. Kliegel, M. A. McDaniel, \& G. O. Einstein (Eds.), Prospective memory: Cognitive, neuroscience, developmental, and applied perspectives (pp. 115-140). Mahwah, NJ: Erlbaum.

Lewandowsky, S. (2011). Working memory capacity and categorization: Individual differences and modeling. Journal of Experimental Psychology: Learning, Memory, and Cognition, 37(3), 720-738.

Logie, R. H., Cocchini, G., Delia Sala, S., \& Baddeley, A. D. (2004). Is there a specific executive capacity for dual task coordination? Evidence from Alzheimer's disease. Neuropsychology, 18(3), 504 513.

MacLeod, C., \& Donnellan, A. M. (1993). Individual differences in anxiety and the restriction of working memory capacity. Personality and Individual Differences, 15(2), 163-173.

Mahy, C. E., Kliegel, M., \& Marcovitch, S. (2014). Emerging themes in the development of prospective memory during childhood. Journal of Experimental Child Psychology, 127, 1-7.

Mahy, C. E. V., \& Moses, L. J. (2011). Executive functioning and prospective memory in young children. Cognitive Development, 26, 269-281.

Mahy, C. E., Moses, L. J., \& Kliegel, M. (2014). The development of prospective memory in children: An executive framework. Developmental Review, 34(4), 305-326.

Mahy, C. E., \& Munakata, Y. (2015). Transitions in executive function: Insights from developmental parallels between prospective memory and cognitive flexibility. Child Development Perspectives, 9(2), $128-132$.

Mărcuș, O., Stanciu, O., MacLeod, C., Liebregts, H., \& Visu-Petra, L. (2015). A FISTful of emotion: Individual differences in trait anxiety and cognitive-affective flexibility during preadolescence. Journal of Abnormal Child Psychology, 1-12.

Marsh, R. L., Hancock, T. W., \& Hicks, J. L. (2002). The demands of an ongoing activity influence the success of event-based prospective memory. Psychonomic Bulletin and Review, 9, 604-610. 
Marsh, R. L., \& Hicks, J. L. (1998). Event-based prospective memory and executive control of working memory. Journal of Experimental Psychology: Learning, Memory, and Cognition, 24, 336-349.

McDaniel, M. A., \& Einstein, G. O. (2000). Strategic and automatic processes in prospective memory retrieval: A multiprocess framework. Applied Cognitive Psychology, 14, 127-144.

McDaniel, M. A., Guynn, M. J., Einstein, G. O., \& Breneiser, J. (2004). Cue-focused and reflexive-associative processes in prospective memory retrieval. Journal of Experimental Psychology: Learning, Memory, and Cognition, 30(3), 605-614.

McNerney, M. W., \& West, R. (2007). An imperfect relationship between prospective memory and the prospective interference effect. Memory \& Cognition, 35(2), 275-282.

Nauta, M. H., Scholing, A., Rapee, R. M., Abbott, M., Spence, S. H., \& Waters, A. (2004). A parent-report measure of children's anxiety: Psychometric properties and comparison with child-report in a clinic and normal sample. Behaviour Research and Therapy, 42(7), 813839.

Ng, E. L., \& Lee, K. (2010). Children's task performance under stress and non-stress conditions. Cognition \& Emotion, 24(7), 1229-1238.

Nigro, G., Brandimonte, M. A., Cicogna, P., \& Cosenza, M. (2014). Episodic future thinking as a predictor of children's prospective memory. Journal of experimental child psychology, 127, 82-94.

Owens, M., Stevenson, J., Norgate, R., \& Hadwin, J. A. (2008). Working memory partially mediates the relationship between trait anxiety and academic performance. Anxiety, Stress, \& Coping, 21, 417-430.

Rosenbaum, P. R., \& Rubin, D. B. (1983). The central role of the propensity score in observational studies for causal effects. Biometrika, $70(1), 41-55$.

Smith, R. E. (2003). The cost of remembering to remember in eventbased prospective memory: Investigating the capacity demands of delayed intention performance. Journal of Experimental Psychology: Learning, Memory, and Cognition, 29, 347-361.

Smith, R. E. (2008). Connecting the past and the future: Attention, memory, and delayed intentions. In M. Kliegel, M. A. McDaniel, \& G. O. Gilles (Eds.), Prospective memory: Cognitive, neuroscience, developmental, and applied perspectives (pp. 27-50). Mahwah, NJ: Erlbaum.
Smith, R. E. (2010). What costs do reveal and moving beyond the cost debate: Reply to Einstein and McDaniel (2010). Journal of Experimental Psychology: Learning, Memory, and Cognition, 36(4), 1089-1095.

Smith, R. E., \& Bayen, U. J. (2004). A multinomial model of event-based prospective memory. Journal of Experimental Psychology: Learning, Memory, and Cognition, 30(4), 756-777.

Smith, R. E., \& Bayen, U. J. (2005). The effects of working memory resource availability on prospective memory: A formal modeling approach. Experimental Psychology, 52(4), 243-256.

Smith, R. E., Hunt, R. R., McVay, J. C., \& McConnell, M. D. (2007). The cost of event-based prospective memory: Salient target events. Journal of Experimental Psychology: Learning, Memory, and Cognition, 33(4), 734-746.

Steinberg, L. (2005). Cognitive and affective development in adolescence. Trends in Cognitive Sciences, 9(2), 69-74.

incaş, I., Cheie, L., Mocan, O., Benga, O., \& Visu-Petra, L. (2016). Validating the Revised Child Anxiety and Depression Scale on the Romanian population.

Visu-Petra, L., Cheie, L., Benga, O., \& Alloway, T. P. (2011). Effects of trait anxiety on memory storage and updating in young children. International Journal of Behavioural Development, 35(1), 38-47.

Visu-Petra, L., Cheie, L., \& Mocan, O. (2013). Executive functioning in high-trait anxious children: A cognitive vulnerability factor? Stress and Anxiety: Applications to Health and Well-Being, Work Stressors, and Assessment, 153-160.

Wang, L., Kliegel, M., Liu, W., \& Yang, Z. (2008). Prospective memory performance in preschoolers: Inhibitory control matters. European Journal of Developmental Psychology, 5(3), 289-302.

Weissman, M. M., Wolk, S., Wickramaratne, P., Goldstein, R. B., Adams, P., Greenwald, S.,...\& Steinberg, D. (1999). Children with prepubertal-onset major depressive disorder and anxiety grown up. Archives of General Psychiatry, 56(9), 794-801.

West, R., \& Bowry, R. (2005). Effects of aging and working memory demands on prospective memory. Psychophysiology, 42, 698-812.

West, R., Bowry, R., \& Krompinger, J. (2006). The effects of working memory demands on the neural correlates of prospective memory. Neuropsychologia, 44, 197-207. 